\title{
TOBACCO ADVERTISEMENTS TARGETED ON WOMEN: CREATING AN AWARENESS AMONG WOMEN
}

\author{
Dorota Kaleta', Bukola Usidame ${ }^{1}$, Kinga Polańska² \\ 'Department of Preventive Medicine, Medical University, Łódź, Poland \\ ${ }^{2}$ Department of Environmental Epidemiology, Nofer Institute of Occupational Medicine, Łódź, Poland
}

\begin{abstract}
SUMMARY
It has been always believed that men smoke more than women, but the trend of smoking in women is increasing nowadays. In some countries there are even more female smokers than male smokers. This is a major health risk because women are present and future mothers, and increasing number of smoking women will enlarge the number of exposed children. Relatively few women are aware of gender-specific health risks, including cervical cancer, osteoporosis, poor pregnancy outcome and early menopause. Tobacco related diseases are on the rise in women, considering the fact that more women now die of lung cancer than breast cancer. Tobacco companies have invented various ways to target women through tobacco advertising despite the various bans. This inevitably leads to the increase in female smoking rates. There are various recommendations from the World Health Organization which include the need for governments to pay particular attention to protect women from the tobacco companies' attempts to lure them into lifetimes of nicotine dependence and to take up counter advertisements against the tobacco companies.
\end{abstract}

Key words: tobacco advertisements, women, smoking, World Health Organization

Address for correspondence: D. Kaleta, Department of Preventive Medicine, Medical University, Żeligowskiego 7/9, 90-752 Łódź, Poland. E-mail: dkaleta@op.pl

\section{INTRODUCTION}

The global prevalence of tobacco use is substantially higher in men $(47 \%)$ than in women $(12 \%)$, reflecting the traditionally low prevalence of female smoking in many developing countries. However, the tobacco industry has targeted women in promotional strategies, calculating that the large population of women who do not use tobacco represent a vast untapped market for tobacco (1). According to the World Health Organization (WHO), women comprise about $20 \%$ of the world's more than 1 billion smokers. The female population in developing countries will rise from the present 2.5 to 3.5 billion by 2025 , so even if the prevalence remains low, the absolute numbers of women smokers will increase. This makes women a target group for the tobacco industry (2). About 250 million women in the world are daily smokers (about $22 \%$ of women in developed countries and $9 \%$ of women in developing countries). In addition, many women in south Asia chew tobacco. Cigarette smoking among women is declining in many developed countries, notably Australia, Canada, the UK and the USA, but this trend is not found in all of those countries. In several southern, central and eastern European countries cigarette smoking is either still increasing among women or has not shown any decline (3). Additionally in some Nordic and western European countries there is little or no difference between male and female smoking rates or as it is in Sweden the percentages of daily smokers (among age group 16-84 years) is even higher among women than men (16\% vs. $12 \%$ in 2007) (4).

Table 1 presents data of daily cigarette smoking for selected European countries. The results of a national survey conducted in Poland, in 2007 , indicated that $23 \%$ of women smoked daily, $3 \%$ were occasional smokers, $10 \%$ were former smokers and $64 \%$ never smoked (5). Additionally, data from 2000 indicated that tobacco-smoking has caused 69,000 deaths in Poland of which $12,000(17.4 \%)$ were in women and $6,000(50 \%)$ of them were premature deaths (at age 35-69 years) (5-6). In the early 1980s, the frequency of smoking increased particularly among women, to about $30 \%$ for daily smokers, driven, among other things, by the introduction of cigarette vouchers and the rationing of tobacco products. In 1982, the percentage of smokers was the highest in the country's history and one of the highest in Europe. That percentage reached $70 \%$ among young and middle-aged men, and 50\% in similar-aged women (5). Between 1990 and 1998, the percentage of girls smoking cigarettes increased among 13-year-olds from $3 \%$ to $10 \%$ and among 15 -year-olds from $16 \%$ to $28 \%$. It seems that the phenomenon was at least partially related to the mass advertising of cigarettes aimed primarily at adolescent girls and young women (5). In the 1990s, social acceptance of smoking noticeably lessened. Comparative international studies conducted in Poland and EU countries showed that in the late 1990s the social climate against smoking in Poland was the strongest in Europe. The change in attitude towards smoking was stimulated by population-based public awareness campaigns, smoking cessation programmes, effective enforcement of comprehensive tobacco control legislation and the launch of a government programme aimed at reducing smoking and its health consequence. The number of people who gave up smoking went into millions and a clear fall in smoking incidence among smokers of both sexes was observed (5). Among men, the fall in percentage of daily 
smokers occurred in all age groups (from around 60\% in 1982 to $40 \%$ in $2000-2004$ ), reaching among all men approximately $1 \%$ annual rate of decline. Among women the highest decline in incidence of daily smoking occurred in the youngest age group (20-29 years: from $50 \%$ to $25 \%$ ). Subsequent surveys in 2002 and 2006, conducted after the enforcement of a complete ban on advertising, promotion and sponsorship of tobacco products, show that smoking prevalence among girls leveled off and subsequently began to fall (6-7).

On the other hand, smoking trends in Ukraine has been observed to be increasing in recent years despite the fact that the public and the authorities have become more aware of the harm related to tobacco and the need for tobacco control measures. In 2005, the Law of Ukraine on Measures to Prevent and Reduce the Use of Tobacco Products and their Harmful Impact on the Health of the Population was adopted (called "The tobacco control law"). In 2006, Ukraine ratified the WHO Framework Convention on Tobacco Control (WHO FCTC), which is a binding international agreement (8-9). The cross-sectional surveys conducted in all regions of the country found a considerable increase in smoking prevalence between 2001 and 2005 (9). In Ukraine an average of additional $1.5-2 \%$ of adult women (aged 15+ years) and 3-4\% of adult men were taking up smoking each year. In a survey done in $2001(18+)$ with a sample size of about 2000 people, $31 \%$ of them were smokers and $10 \%$ where female smokers.

Another survey done in 2005 (including children from age $15-17$ years) indicated that $41 \%$ of the population was current smokers and $20 \%$ was female current smokers. The smoking prevalence in women aged $>18$ years increased from $4.9 \%$ of the population (18.8\% of total smokers) in the year 2000 to $6.4 \%$ in the year 2006 (23.3 of total smokers) (9-10). The upward trend in smoking prevalence has been linked to the simultaneous fall in the real prices of cigarettes. The characteristics of the tobacco epidemic among Ukrainian women suggest that it may be related to the processes of emancipation and social improvement. Women are more likely to smoke if they are young, live in large cities and have a university education. The epidemic is, however, now spreading more widely: women in older age groups and those living in smaller settlements are showing increasing trends towards becoming smokers, although a university education remains associated with the highest risk of smoking uptake. The epidemiological study conducted by the Institute of Cardiology of Academy of Medical Sciences of Ukraine revealed that within the last 25 years, the smoking rate among women increased 3 -fold in urban areas and 17-fold in rural areas (9).

As the other example in Slovakia in 2003 about $31 \%$ of the adult population were current smokers and $23 \%$ were female adult current smokers, a total ban on tobacco advertising in the electronic media (TV and radio), in print media (daily and weekly papers and magazines on the national, regional and local level) and in outdoor advertising (billboards, airbuses, etc.) was imposed $(4,11)$. Due to the effective total ban on tobacco advertising and the restriction on financial sources to be used on tobacco advertising, the tobacco business was forced to focus on sponsorship through gift-receivers, sponsorship on events focused on children and youth education (e.g.: be cool, be in, etc.) and sport events, culture and art - sponsorship of the national cultural institutions (national level), protection of the environment - (regional and local level), active presence in various events in order to acquire
Table 1. Comparison of daily cigarette smoking in selected European countries (data sources: WHO Report on the Global Tobacco Epidemic, 2009)

\begin{tabular}{|l|c|c|}
\hline \multirow{2}{*}{ Country (year) } & \multicolumn{2}{|c|}{ \% of daily tobacco smokers } \\
\cline { 2 - 3 } & women & men \\
\hline Austria (2004) & 35.5 & 40.2 \\
\hline Czech Republic (2008) & 19.3 & 26.1 \\
\hline Germany (2005) & 18.8 & 27.9 \\
\hline Greece (2002) & 39.0 & 47.4 \\
\hline Hungary (2003) & 27.7 & 38.6 \\
\hline Poland (2007) & 23.0 & 34.0 \\
\hline Slovakia (2003) & 14.3 & 32.8 \\
\hline Sweden (2007) & 16.0 & 12.0 \\
\hline Ukraine (2005) & 16.7 & 62.3 \\
\hline
\end{tabular}

new young employees for tobacco business (job fairs, etc.) and charitable events for handicapped people (11-12).

According to the data from the Czech Smoking Prevalence Survey (2008) in the Czech Republic, daily tobacco smoking was declared by $22.6 \%$ of the population (age group $15+$ years) and by $19 \%$ of women (4). Additionally, it is important to notice that there has been a decrease of about $36 \%$ in cigarette consumption from 1993 until now. In Austria, in 2004, 47\% of the adult population was current smokers, and the same percentage corresponded to female adult current smokers. Very high percentages of female current smokers were also indicated in Greece and Hungary (40\% and $31 \%$ respectively) (4). In Germany, those percentages were lower ( $27 \%$ for total population and $22 \%$ for women) (4). About 8 out of every 75 deaths in Germany are attributed to smoking related diseases for women between the age of 35-69. For women over the age of 70, eighteen out of every 369 deaths are attributed to smoking related diseases $(10,13)$. Ban on tobacco smoking in Germany is placed in just a few measures including advertising to certain audiences and smoking on buses (8).

Contrary to the belief that almost everyone is aware of the health risks associated with tobacco, one British survey found that nearly $99 \%$ of women did not know of the link between smoking and cervical cancer. Another survey found that $60 \%$ of Chinese adults did not know that smoking can cause lung cancer while $96 \%$ were unaware it can cause heart disease $(14,15,16)$. In 1923 , when lung cancer was extremely rare, women consumed only $5 \%$ of all cigarettes sold. By 1929, it was up to $12 \%$ and by 1935 , $18 \%$. It peaked at $33 \%$ in 1965 and remained virtually unchanged through 1977. The themes of tobacco marketing to women typically associate smoking with feminism, sophistication, weight control, and Western-style independence. The rapid increase in smoking prevalence that occurred among women in Spain, Italy, and Greece since the 1970s, decades after the increase in female smoking in other Western countries, demonstrates that cultural prohibitions against tobacco use by women can be eclipsed by aggressive tobacco marketing aimed at women. In the European School Survey Project on Alcohol and other Drugs (ESPAD) survey of 15-16 year old school children done in 1999, girls were more likely than boys to report having smoked at least 40 times in their lifetime in eight out of 23 participating countries 
(Bulgaria, Denmark, Ireland, Italy, Malta, Norway, Slovenia, and the United Kingdom) (1).

Since the advent of the Tobacco Control Initiative by the WHO, there has been a ban in various countries at different levels regarding the use of tobacco in public places and advertising of tobacco products $(7,12-13)$. Despite the ban, the tobacco industries have found a variety of ways to keep pushing their brands with subtle advertisements to women. Women represent a fresh and lucrative target for the tobacco industry. A recent study from the WHO denotes selling tobacco products to girls and women the single largest marketing opportunity in the world (3). In 1996, only between $2 \%$ and $10 \%$ of women in developing countries smoked, compared to $25 \%$ to $30 \%$ of women in developed countries, causing them to recruit smokers from emerging population. The target regions for tobacco companies include Asia, Africa, India and former Soviet bloc nations - areas in which the rates of smoking among women are far lower than for men. Data indicate that tobacco smoking in girls and women in the European region are catching up with the smoking prevalence in men, or in some cases are even more $(1,4)$. WHO estimates that there are currently 4 million deaths a year from tobacco, a figure expected to rise to more than 8 million by 2020 . By that date, 70 percent of those deaths will occur in developing countries $(13,15)$. Tobacco companies have to recruit about 4,000 new smokers daily just to maintain their current market size. Even that global estimates indicate the higher prevalence of smokers among men comparing to women, the epidemic of tobacco use is rising rapidly among women, particularly young women making them the target group for tobacco industry.

The aim of the paper was to analyze the reasons and the methods used by tobacco industry to target the women population on one hand and the action taken at the international and national level to protect them on the other.

\section{MATERIALS AND METHODS}

We basically made use of most of the WHO documents in relation to prevalence and trends in smoking among women and tobacco advertising targeted to women population. Additionally Medline, PubMed and Ebsco search were performed to identify the studies focusing on women, tobacco consumption, ban of advertising and the methods used by tobacco industry to target women. Only English papers were considered. The following keywords were used: smoking, women, advertising, tobacco industry, advertising ban, ban on promotion and sponsorship.

\section{RESULTS}

\section{Various Reasons for Targeting Women}

Smoking behaviour of women differ from that of men. Women are more highly motivated to smoke, they find it harder to stop smoking and they are also more neurotic than men. There are various reasons why women are a lucrative target for the tobacco industry including the fact that girls' and women's spending power is increasing so that cigarettes are becoming more affordable. Additionally, changing norms may put more women at risk of smoking. Greater female autonomy and changes in women's roles are associated with smoking uptake in many countries. The social and cultural constraints which previously prevented many women from smoking, such as in China and in Muslim countries, are weakening. In addition, women-specific health education and quitting programmes are rare, especially in developing countries. The governments in developing countries may also be less aware of the harmful effects of tobacco use for women as they mostly see tobacco as a problem confined to men $(16,17)$. Examples of the main reasons for initiation of tobacco use in India in various forms were: accepted norms and beliefs and use the tobacco as a medicinal aid (to cure toothaches, during labor). The reason cited for maintenance was dependence. Women may be especially prone to nicotine addiction as studies have shown that distinct gender differences increase their vulnerability to addiction (18).

There is a common misconception that smoking helps to relieve stress. Stress was cited many times as an important factor in initiating and continuing smoking as well as returning to smoking among those who have given up (17). Additionally, women smokers may be more afraid than men that they will gain weight if they stop smoking and there is also an evidence that they can experience more severe signs of nicotine withdrawal than men (18).

\section{Methods Used by the Tobacco Industry to Target Women}

The tobacco companies are targeting women with well-funded, alluring marketing campaigns (17). This includes a wide spectrum of activities including product development, distribution, pricing and promotion (18). In developing countries they cleverly link the emancipation of women with smoking, using slogans similar to what was used in western countries decades ago, such as "You've come a long way, baby" $(16,19)$.

Cigarettes advertised with false images of emancipation, stylishness, sophistication, sexual allure, mistaken belief that smoking assists with weight loss and the misperception created by the tobacco industry that smoking enhances social popularity $(16,17)$. The message that smoking helps stay slim appeared very early in the USA with the infamous ad of the 1930s encouraging women to reach for a cigarette "instead of a sweet" (20). This message continues to be promoted today, although more subtly, through the association of slender female models with slender cigarettes (17).

Easy access to tobacco products, low prices, peer pressure, tobacco use and approval by peers, parents and siblings and female oriented media are other factors (17). The tobacco industry also introduced the use of "low tar" and "light" cigarettes to women realizing that many of them were concerned about the long-term health risks of smoking and creating false image that those cigarettes are "softer" or even "safer" (17). Although in many countries the usage of those terms is banned the tobacco brands use the same shape and colors which can be easily linked to the previously used terms.

Tobacco industries market a variety of cigarette brands to girls and women indicating "female only" (17). Perfumed scents, exotic flavours and attractive packaging make the cigarettes desirable for women especially for the young ones $(17,19)$.

Cigarette brands for younger women stressed female camaraderie, self confidence, freedom, and independence; cigarette brands for older women addressed needs for pleasure, relaxation, social acceptability, and escape from daily stresses (21). 


\section{Exploitations of the Tobacco Industry: Methods Being Used by Tobacco Companies Despite Ban}

From the history of advertising and women's smoking, it has been researched that the social stigma attached to women smoking has grown over the years. As late as 1908 a woman in New York was arrested for smoking a cigarette in public, and in 1921 a bill was proposed in the US Congress to ban women from smoking in the District of Columbia. It is therefore remarkable that within 50 years of the invention of the mass produced cigarette, smoking among women in North America and northern Europe has become socially acceptable and even socially desirable. This can be attributed to the way in which the tobacco industry capitalised on changing social attitudes towards women by promoting smoking as a symbol of emancipation, a "torch of freedom" with the aid of judicious marketing, into a socially acceptable and desirable practice (22).

There have been various kinds of bans and restrictions on tobacco advertisements. The countries have enforced a complete ban on advertising on national and cable television and radio (there is no ban on cable television in Serbia). Fifteen countries have enforced a total ban in local printed media, 14 countries for billboards, 11 countries in cinemas and 5 countries at the point of sale and kiosks. The rest either have partial bans or no restrictions $(4,23)$. As the example in 2003, the Law of Ukraine on Advertising was changed to include new amendments regarding tobacco advertising in addition to television and radio. Tobacco was banned on transport vehicles, indoors and through advertising activities, prohibited within 300 meters from educational institutions for minors and in printed media aimed at young people. There was a ban on tobacco industry sponsorship for television and radio programmes, sporting and other events. Outdoor tobacco advertising was to be banned from 2009 and in the print media from $2010(9,12)$. There is also a broad ban on tobacco advertising and marketing in Poland. Tobacco is banned on radio and television, print media, electronic media, billboards. The ban also encompasses the promotion of tobacco products and sponsorship by tobacco companies of sport, cultural, educational, health, social and political activities. In 2010, Poland's acting president signed a new law that bans smoking in many public places (24). In the Czech Republic, tobacco advertising has been banned in local printed media, on bill boards, cinemas, television and radio. All forms of sponsorship by tobacco companies are also banned (4). In Hungary this ban covers the press, radio and the internet. Further, cigarette manufacturers are not allowed to sponsor important cultural or sporting events (23).

What has been however noted, was that advertising remains cardinal to the tobacco industry. The role and importance of these 'other' media is growing as advertising restrictions increase. Based on this, many companies also give out free cigarette samples and sponsor cultural and athletic events in countries where tobacco advertising is restricted or severely limited. The particular marketing strategy used to target women vary on factors such as: the tobacco company, the country, its culture, smoking patterns and trends, and its restrictions on advertising and promotion. It is also important whether the campaign is aimed at a particular subgroup of women, defined by characteristics such as age, ethnicity and affluence. Tobacco advertising has been dominated by three themes: providing satisfaction (taste, freshness, mildness, etc.), assuaging anxieties about the dangers of smoking, and creating associations between smoking and desirable outcomes (independence, social success, sexual attraction, thinness, etc. (21).

In many countries, particularly where tobacco advertising is banned from television, the most popular medium for targeting women is women's magazines. Cigarette advertising in these magazines has grown substantially (25-27). In the USA, eight of the twenty magazines receiving the most cigarette advertising are women's magazines. In the United Kingdom, revenue from tobacco ads in women's magazines increased by $50 \%$ in real terms between 1977 and 1982, and by 10\% between 1984 and 1988. In 1988, nearly 9.7 million pounds was spent on cigarette advertisements compared with 7.2 million in 1984. A survey of the top women's magazines in 14 European countries found that $72 \%$ of them accepted cigarette advertisements (28-29). There is also evidence that these media outlets report very little about the health hazards of smoking. The creation of brand personality was to be achieved using aspects of style, tone, music and visuals, rather than information, for the creative team believed that in cigarette advertising $90 \%$ of what you communicate is non-verbal (26). There are several reasons why women's magazines are so popular with tobacco advertisers. Women's magazines represent a battleground between the competing interests of, on one hand, the tobacco companies and advertisers wishing to target their massive female audience and the magazines wishing to maximize their own revenues, and, on the other, editors and journalists wishing to report the fact that without bias and to protect the health of their readers. At present, the tobacco industry seems to be winning the battle.

Women are targeted through a variety of other promotional activities as well. These have included special offers such as free silk stockings, contests, free cosmetics, and clothes or towels carrying the brand logo. Another popular method is the sponsorship of women's sports (e.g. tennis) which receive widespread media coverage, and of women's events such as fashion shows, the use of branded items such as sweaters and jerseys. Again, the aim is to enhance the brand's image by associating it with social activities (17, 26-27).

The industry has sponsored fashion shows in shopping malls linked to magazine promotions in the USA. Such sponsorship and public smoking by super-models and film stars associates tobacco with glamour (29).

The companies and their advertising agencies used an array of terms to refer to indirect advertising such as "parallel communication", "alibi advertising", "logo licensing", "image transfer advertising", and "below the line advertising". A Hong Kong advertising executive described the difference: "advertisements (above the line) give the image, below the line activities such as sponsorship of athletics or concerts, sell the product" (20). Surveys reported various women's organizations receiving money from tobacco companies to protect their interest. As rightly said, the tobacco industries were aiming to buy 'Innocence by Association'.

\section{Global Efforts by Women to Combat Tobacco Use}

The WHO FCTC highlighted the need for women to participate in the development and implementation of tobacco control policies (8). Tobacco control should aim to improve 
gender-distributive policies that recognize women's exclusion and disadvantage in terms of access to social and economic recourses, as well as decision-making. It is pointed that women must be involved in research and programme planning. Additionally women need to support one another and encourage younger generations to be aware and active in preventing tobacco use among women and girls (18). Number of international and national organizations (including women's organizations) need to be involved in antismoking activities through campaigns, outdoor advertising and the media amongst others. The greatest challenge facing women's organizations is to galvanize the leadership to prevent a rising epidemic of tobacco use among women, particularly young women, before it starts (7). To be successful, women's groups involved in tobacco control programmes have argued that it is necessary to start from girls' and women's own experiences and take into account broader context of women's lives. This is possible when women's leadership is prominent within tobacco control. Women's organizations should be involved in tobacco control because working with women's groups helps to reach other groups, such as husbands and partners, and children, to influence their behaviour and reduce their exposure to environmental tobacco smoke $(2,18)$. Moreover, working with women's organizations can widen the political support for tobacco control, taking it beyond the health community. This may be particularly important when seeking support to introduce specific legislative or regulatory mechanisms. Women leaders offer expertise on women's perspectives and experiences, particularly in networking and building alliances. In recent years some progress has been made in mobilizing nongovernmental organizations (NGOs), foundations, and the scientific community in support of activities concerned with gender, women, and tobacco. For example, the International Network of Women Against Tobacco (INWAT), founded in 1990 by women tobacco control leaders to address the complex issues of tobacco use among women and young girls (30). INWAT is a network of over 1800 members in 100 different countries working toward the elimination of tobacco use and exposure among women. INWAT regularly distributes reports and newsletters and in 2006 published Turning a New Leaf: Women, Tobacco and the Future. Furthermore INWAT provides contacts between individuals and organizations, primarily women, working in tobacco control, shares strategies on countering tobacco advertising and promotion. This Network also supports the development of womencentred tobacco control prevention and cessation programmes and promotes female leadership as well.

Other examples of women to be tobacco-free advocates comes from Sweden. All Miss Sweden contestants must be non-smokers and part of their responsibilities involves visiting schools as role models to educate 10-14 year-olds on the importance of not smoking (18).

In the 1990s and 2000s, a Non Smoking Generation (Sweden) launched several media campaigns about smoking and the use of snus targeting young women in Sweden. The campaigns have included different types of advertising (outdoor, internet) with shocking and controversial messages. The Non Smoking Generation partnered with a popular women's magazine and a famous model agency. All these campaigns are implemented to contribute to reduce the uptake of tobacco and promoting cessation.

\section{CONCLUSIONS AND RECOMMENDATIONS}

The prevalence of smoking by women and the corresponding statistics on tobacco-induced diseases in women serve as a testimony to the success of targeted tobacco advertising. The tobacco industry has been particularly adept at understanding, predicting and capitalizing on the feminist movement by seizing the symbols of feminism and equating women's liberation with smoking.

Research has shown a negative correlation between the amount of tobacco advertising in women's magazines and the number of articles that address the health hazards of tobacco. Tobacco use could kill one billion people during this century. Recognizing the importance of reducing tobacco use among women, and acting upon that recognition, would save many lives. The key rationales cited for implementing a comprehensive ban on tobacco advertising and promotion include the health consequences of tobacco use, the deceptive or misleading nature of several tobacco promotional campaigns, the unavoidable exposure of youth to these campaigns, the role of tobacco advertising and promotion in increasing tobacco use in the population, especially among youth, the targeting of "at-risk" populations, including youth, women, and ethnic and racial minorities, through advertising and promotion, the failure of the tobacco industry to effectively self-regulate its marketing practices; and the ineffectiveness of partial advertising bans.

According to the WHO, a comprehensive ban on all advertising, promotion and sponsorship protects people from industry marketing tactics and could decrease tobacco consumption by about $7 \%$, independent of other tobacco control interventions. Complete bans block the industry's ability to continue marketing to young people who have not yet started to use tobacco, and to adult tobacco users who want to quit. Partial bans have little or no effect taking into account that if advertising is prohibited in a particular medium, the tobacco industry merely redirects expenditures to places where advertising is permitted. The tobacco industry strongly opposes marketing bans because they are highly effective in reducing tobacco use. More articles have to be included in the various women's magazines regarding their health and tobacco use. Substantial efforts are underway to strengthen the infrastructure of global tobacco surveillance through the regional WHO offices. Local tobacco control advocates at the grassroots level provide the underlying momentum for this work. Training programs led by the Centers for Disease Control and Prevention (CDC) and WHO have greatly improved the quality, standardization, and potential availability of data on tobacco use by adolescents. The expertise developed to collect, analyze, and report data on youth tobacco use will facilitate the collection and analysis of other kinds of surveillance data. The development of strong and effective partnerships within countries will strengthen the local capacity for tobacco control. Passage of the draft WHO Framework Convention on Tobacco Control will provide continuing opportunities for grassroots involvement in monitoring overt and covert violations of the convention regarding tobacco marketing. Much more needs to be done in every country including embracing the WHO MPOWER package of six proven policies introduced to help fight the tobacco epidemic. In monitoring tobacco use and prevention policies data gotten is vital in ensuring the success of the other policy interventions. It will help the leaders of the government understand the problems caused 
by tobacco and effectively manage and improve interventions. Second-hand smoke exposure increases the risk of coronary heart disease by $25-30 \%$ and the risk of lung cancer in non-smokers by $20-30 \%$. There is no safe level of exposure to second-hand smoke. Smoke free laws can cut absolute smoking prevalence by $4 \%$. Smoke free policies in several industrialized countries have reduced total tobacco consumption among workers by $29 \%$. The effectiveness of smoke-free environments produces positive health effects in the country. Countries' health care systems hold the primary responsibility for treating tobacco dependence including simple medical advice in respect of pharmacotherapy and quit lines. Comprehensive warnings about the dangers of tobacco are critical to change its image, especially among adolescents and young adults. People need to associate tobacco with its extreme addictiveness and dangerous health consequences, and social undesirability. Government and civil societies can take action by creating policies mandating health warnings on tobacco packages, anti-tobacco counter-advertising campaigns and counter-advertising in media. Complete blockage of all categories of marketing and promotion. A 70\% increase in the price of tobacco could prevent up to a quarter of all smoking-related deaths worldwide. It also directly benefits governments through increased revenues, which can be used for tobacco control and other important health and social programmes.

\section{REFERENCES}

1. Shafey O, Dolwick S, Guindon GE, editors. Tobacco control country profiles. Atlanta: American Cancer Society; 2003.

2. World Health Organization. Tobacco Free Inniciative (TFI): World Health No Tobacco Day, 31 May 2010 [Internet]. WHO: Geneva; 2010 [cited 2010 Nov 15]. Available from: http://www.who.int/tobacco/wntd/2010/ en/.

3. Mackay J, Eriksen M, Shafey O. The tobacco atlas. 2nd ed. Atlanta: American Cancer Society; 2006.

4. World Health Organization. WHO report on the global tobacco epidemic, 2009: implementing smoke-free environments. Geneva: WHO; 2009.

5. World Health Organization. The current status of the tobacco epidemic in Poland. Copenhagen: WHO Regional Office for Europe; 2009.

6. Zatonski W. Democracy and health: tobacco control in Poland. In: deBeyer J, Waverley Brigden LW, editors. Tobacco control policy: strategies, successes and setbacks. Washington: The World Bank; 2003. p. 97-119.

7. Samet JM, Yoon SY, editors. Women and the tobacco epidemic: challenges for the 21st century. Geneva: World Health Organization; 2001.

8. World Health Organization. WHO framework convention on tobacco control. Geneva: WHO; 2003.
9. World Health Organization. Tobacco control in Ukraine: the national report. Copenhagen: WHO Regional Office for Europe; 2009.

10. World Health Organization. World health statistics 2010. Geneva: WHO; 2010.

11. Blazej S. Marketing strategies of the tobacco industry in the Slovak Republic. Proceedings of the 12th World Conference on Tobacco or Health; 2003 Aug 3-8; Helsinky, Finland; 2003.

12. Minh HV, Nga PT. Tobacco use among women: gendered perspective to be included in global tobacco control policies. Indian J Med Res. 2010 May;131:600-2.

13. Shafey O, Mackay J. The tobacco atlas. 3rd ed. Atlanta: American Cancer Society; 2009.

14. World Health Organization. Fact sheets: smoking statistics. Manila: WHO Regional Office for the Western Pacific; 2002.

15. O'Keefe AM, Pollay RW. Deadly targeting of women in promoting cigarettes. J Am Med Womens Assoc. 1996 Jan-Apr;51(1-2):67-9.

16. Frieden TR, Blakeman DE. The dirty dozen: 12 myths that undermine tobacco control. Am J Public Health. 2005 Sep;95(9):1500-5.

17. Amos A. How women are targeted by the tobacco industry. World Health Forum. 1990;11(4):416-22.

18. World Health Organization. Empower women - combating tobacco industry marketing in the WHO European region. Copenhagen: WHO Regional Office for Europe; 2010.

19. World Health Organization. Tobacco control in developing countries. Geneva: WHO; 2000.

20. Toll BA, Ling PM. The Virginia Slims identity crisis: an inside look at tobacco industry marketing to women. Tob Control. 2005 Jun;14(3):17280 .

21. Anderson SJ, Glantz SA, Ling PM. Emotions for sale: cigarette advertising and women's psychosocial needs. Tob Control. 2005 Apr;14(2):127-35.

22. Amos A, Haglund M. From social taboo to "torch of freedom": the marketing of cigarettes to women. Tob Control. 2000 Mar;9(1):3-8.

23. World Health Organization. European tobacco control report 2007. Copenhagen: WHO Regional Office for Europe; 2007.

24. Stafford N. Poland introduces partial ban on smoking in public places. BMJ. 2010 May 5;340:c2428.

25. Assunta M, Chapman S. The tobacco industry's accounts of refining indirect tobacco advertising in Malaysia. Tob Control. 2004 Dec;13 Suppl 2:ii63-70.

26. World Health Organization. Gender, health and tobacco. Geneva: WHO; 2003.

27. Weiser, R. Smoking and women's magazines, 2001-2002. New York: American Council on Science and Health; 2004.

28. Ronald MD, et al. The Role of the media in promoting and reducing tobacco use. Rockville: National Cancer Institute; 2008.

29. Corson J. Heartbeat Wales: a challenge for change. World Health Forum. 1990;11(4):405-11.

30. International network of women against tobacco [Internet]. Vancouver: British Columbia Centre for Excellence in Women's Health; 2011 [updated 2011; cited 2011 March 9]. Available from: http://www.inwat.org/. 